\title{
Super modules-based active QR codes for smart trackability and IoT: a responsive-banknotes case study
}

João F. C. B. Ramalho (iD) ${ }^{1}$, Sandra F. H. Correia ${ }^{1}$, Lianshe Fu ${ }^{1}$, Lília M. S. Dias ${ }^{1}$, Pedro Adão ${ }^{2}$, Paulo Mateus ${ }^{3}$, Rute A. S. Ferreira $\mathbb{B}^{1 凶}$ and $^{\circ}$ Paulo S. André (iD ${ }^{4 凶}$

The general use of smartphones assigns additional relevance to QR codes as a privileged tool to the Internet of Things (loT). Crucial for QR codes is the evolution to loT-connected smart tags with enhanced storage capacity and secure accesses. Using the concept of super-modules (s-modules) built from adjacent spatial multiplexed modules with regular geometrical shapes, assisted by colour multiplexing, we modelled and design a single QR code with, at least, the triple storage capacity of an analogous size black/white QR code, acting as a smart-tag ensuring restrict access and trackability. The s-modules are printed using luminescent low-cost and eco-friendly inks based on organic-inorganic hybrids modified by lanthanides with multiplexed colour emission in the orthogonal RGB space. The access to the restrict information is attained only under UV irradiation and encrypted for secure transmission. The concept of active QR codes for smart trackability and loT was materialised through the development of a free friendly-user mobile app.

npj Flexible Electronics (2020)4:11; https://doi.org/10.1038/s41528-020-0073-1

\section{INTRODUCTION}

Internet of Things (IoT) is expanding daily, reaching applications in the fields of industry ${ }^{1}$ and smart cities ${ }^{2}$, bridging living entities, processes and devices with the Information and Communication Technologies (ICT), inspiring new concepts as "Internet of NanoThings"3, "Internet of Food"4 , "Internet of Biology"5 or "Internet of Animals" ${ }^{\prime \prime}$. Simultaneously, loT promotes remotely data exchange for processing/management, leading to larger and more complex networks ${ }^{7}$. Thus, loT can be considered as an ubiquitous network, which allows the communication between human-to-human $(\mathrm{H} 2 \mathrm{H})$, human-to-machine $(\mathrm{H} 2 \mathrm{M})$ and machine-to-machine (M2M), in which each element has a unique identity ${ }^{8}$. Therefore, tag elements for identification (people or objects) are of paramount relevance.

New smart labels able to real-time sensing and to establish $\mathrm{H} 2 \mathrm{H}$, $\mathrm{H} 2 \mathrm{M}$ and $\mathrm{M} 2 \mathrm{M}$ communication are a growing market busted by applications as Industry 4.0, Smart Cities, and Smart and Connected Communities ${ }^{9}$ that is expected to worth 16.12 billion dollars by 2025 (https://www.grandviewresearch.com/industryanalysis/smart-label-market). In this framework, smart labels for authentication, trackability and counterfeiting detection, with controlled (public, restrict or encrypted) accesses to information are relevant ${ }^{10-12}$. From the user point of view, loT smart labels should be reliable, ensuring the integrity, authenticity and preservation of information for objects in circulation or during transactions ${ }^{13}$.

QR codes are described as a gateway to loT, as its remarkable evaluative process points out. From handwrite codes, the first major evolutive step was the arising of barcodes in $1952^{14}$ that, in 1994 were develop into matrix structures, like QR Codes. The full potential and widespread of QR codes become a reality, in recent years, with the massive use of smartphones incorporating image acquisition modules and enhanced data processing capacities.
The well-known advantages of QR codes such as fast and easy readability independent from the orientation, the ability to endure physical damages recurring to error correction codes, place them as good candidates to be part of the next evaluative step of tag elements. Moreover, compared to electronic labels, QR codes optical elements provide extra advantages such as easy manufacturing (materials based ink printing) $)^{15-20}$ and reading (smartphones) with lower production and operation costs (CAPEX and OPEX), strictly visual and tactile (appealing for societal applications), environmentally sustainable (easy recycling), among others.

Here, we propose a new approach to the design and implementation of an improved identification optical tag that meets the challenges of QR codes and covers the demanding features of smart labels. In particular, QR codes based on spatial and colour multiplexed super-modules (s-modules) able to increase the storage capacity of a single QR code up to, at least, three times, using the RGB colour orthogonal space s1-23 $^{21}$ are modelled and fabricated. The $s$-modules printed with eco-friendly luminescent inks form distinct layers of information storage with the public, restrict and/or encrypted accesses, yielding a new generation of active QR codes materialised in the development of a free access mobile application for smartphones (mobile app).

\section{RESULTS}

Spectrally selective luminescent QR codes

Spectrally selective colour multiplexed luminescent QR codes will be produced with the ability to store information at different layers of accessibility (Fig. 1) allowing the control of the provided information for (i) public access and (ii) restricted access. The public access, available under ambient illumination (Fig. 1a) can be scanned with the user's mobile device and provides a link to an internet server containing static informational details (e.g.,

\footnotetext{
${ }^{1}$ Department of Physics and CICECO - Aveiro Institute of Materials, University of Aveiro, 3810-193 Aveiro, Portugal. ${ }^{2}$ Department of Computer Science and Engineering and Instituto de Telecomunicações, Instituto Superior Técnico, Universidade de Lisboa, 1049-001 Lisbon, Portugal. ${ }^{3}$ Department of Mathematics and Instituto de Telecomunicações, Instituto Superior Técnico, Universidade de Lisboa, 1049-001 Lisbon, Portugal. ${ }^{4}$ Department of Electrical and Computer Engineering and Instituto de Telecomunicações, Instituto Superior Técnico, Universidade de Lisboa, 1049-001 Lisbon, Portugal. ${ }^{\circledR}$ email: rferreira@ua.pt; paulo.andre@lx.it.pt
} 


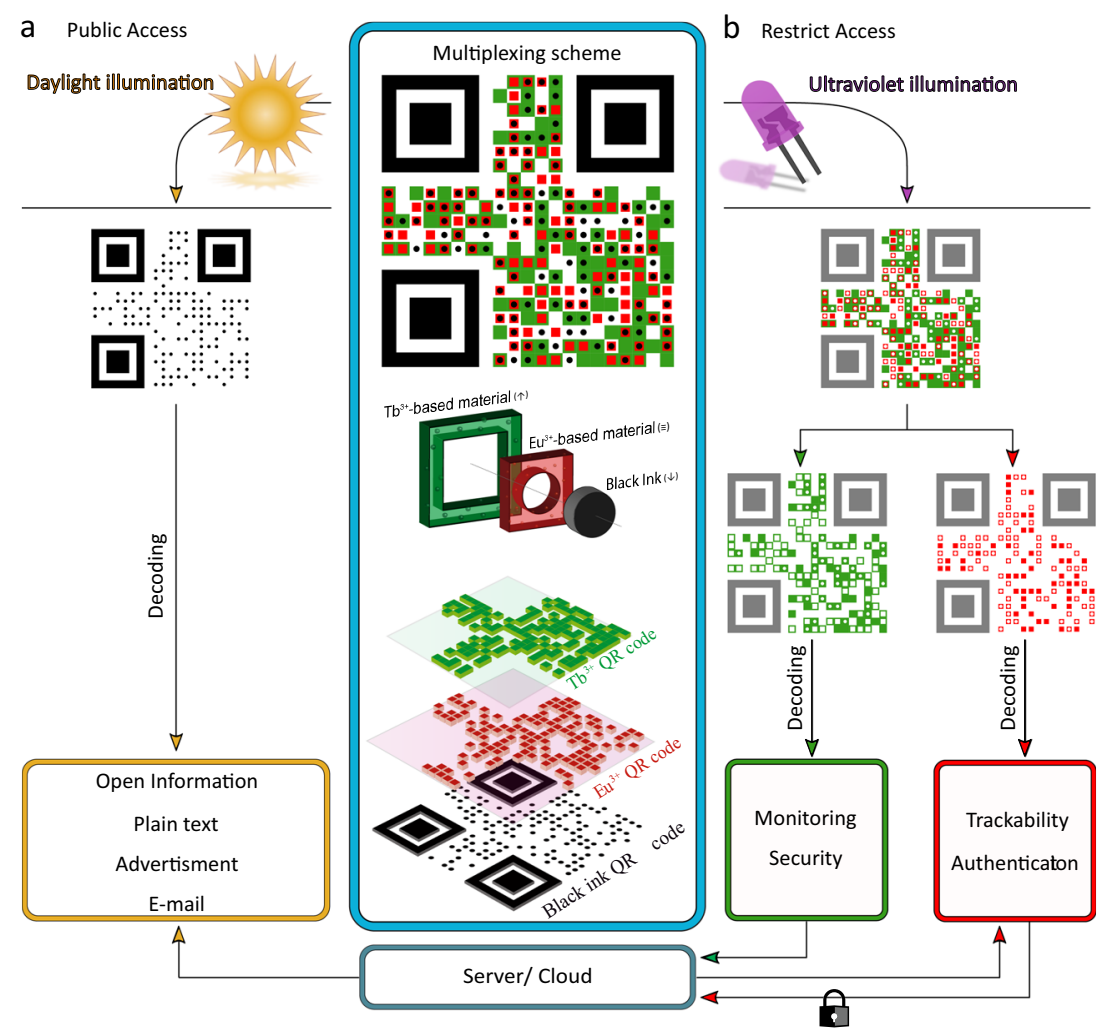

Fig. 1 Schematic of the proposed super-modules and multiplexing process. The QR codes are aggregated into a single one (spatial multiplexing) where the modules fit perfectly one inside the others (s-modules). The QR code contains 3 levels of information due to use of 3 independent inks (colour multiplexing) and shapes, allowing: a public access, read with ambient light, providing information to the user through the applications available at any mobile device and $\mathbf{b}$ restricted access with illumination UV/blue LED, read via dedicated applications/devices, that enable the monitoring and security, triggering alerts thought the establishing of an encrypted connection to a server.

thematic topics, historical content) or dynamic information (e.g., currency exchange rate). The restricted access is granted with illumination from UV/blue light-emitting diodes (LEDs, Fig. 1b) and scanned recurring to dedicated applications. The encrypted messages stored in the restricted access layer, containing unique authentication tags, can be securely transmitted to a remote server, which enables the trackability and the triggering of security alerts or other additional security features.

The colour multiplexing is attained using luminescent materials in the visible spectral region, activated by UV radiation and displaying red $(R)$, green $(G)$ and blue $(B)$ emission ${ }^{24}$, being each colour assigned to a selective s-module-based QR code and related with a layer of accessibility. The demultiplexing process is ensured by the RGB colour space orthogonality. To achieve spectral selectivity (selective excitation energy), luminescent QR codes will be manufactured using flexible and transparent organic-inorganic hybrids materials modified by lanthanide $\left(\mathrm{Ln}^{3}\right.$ ${ }^{+}$)-based complexes. The hybrids are processed at room temperature with the desired shape and thickness, combining the flexibility of the organic counterpart with the mechanical stability of the inorganic one ${ }^{25-27}$. Among the different organicinorganic hybrids, the emphasis is given to di-ureasils, whose siliceous-based skeleton provides compatibility with current microelectronics confers enhanced thermal stability with the onset of the decomposition temperature at $\sim 339^{\circ} \mathrm{C}$ compared with pure polymer-based host ${ }^{28}$. Di-ureasils are processed using environment-friendly green solvents with tuneable viscosity, which makes them ideal low-cost inks to be easily printed in virtually any substrate (paper, plastic, and textile) similarly to what occurred in printable electronic circuits, which will further promote the spread of the IoT applications. Moreover, the possibility of incorporating optically active centres with light emission properties tuned along the visible spectral range is of great advantage, as distinct multiplexing colours may be selected. Therefore, organic complexes of trivalent lanthanide ions $\left(\mathrm{Ln}^{3+}\right.$, $\mathrm{Ln}=\mathrm{Eu}, \mathrm{Tb}$ ) were selected as they are nearly pure red and green emitters, respectively ${ }^{29}$, permitting the hybrids to keep their transparency under daylight illumination.

The active QR codes concept will be assisted by the combination of colour multiplexing and spatial multiplexing through distinct colours coordinates and different module shapes (circle, square and hollow square, Fig. 1), respectively, with optimised dimensions yielding an s-module with the triple storage capacity of an individual one. The layers assigned to each module shape combines perfectly without overlapping avoiding extra misinterpretation (in the demultiplexing step) which is present when colours are mixed due to the modules (of distinct colour) superposition. In this case, the colour multiplexing is used apart from the spatial multiplexing here proposed, which is a clear advantage gained through the s-modules since the result is a single multiplexed QR code with the ability to store the triple capacity compared to conventional black/white ones. The $s$-modules innovative spatial multiplexing ensures itself colour separation that is not affected by the background emission, even when the red and green QR codes are simultaneously active. The red and green colours separation is ensured regardless of the emission intensity since these colours are the base functions (used to describe all the visible colours) for the RGB orthogonal colour space, thus linearly independent between them, never mixing, and being discerned one from another. We also note that other colour spaces establish red and green colours as complementary ones $^{30}$, thus, also in this case, the easiest colour to be discerned 
from red is green and vice-versa. To unequivocally demonstrate the potential of luminescent QR codes as smart optical tag elements for security and real-time monitoring, we select, as an example, the banknotes trackability application.

To reveal the luminescent $\mathrm{QR}$ codes two distinct excitation wavelengths, UVC ( $254 \mathrm{~nm})$ and UVA ( $365 \mathrm{~nm})$, were selected. This methodology to activate colour in the banknotes is identical to that used nowadays to detect safety elements in the current money. Optically activated features are already used in banknotes, for instance, in the Euro banknotes (see the European Central Bank website for additional information, https://www.ecb.europa.eu/ euro/banknotes/security). For Euro banknotes, under standard UVA irradiation, small fibbers embedded in the paper present yellow, green and red colour emission, enlightening special areas of the banknote, and under UVC yellow and orange colours are displayed (Supplementary Fig. 1). The use of UV-activated luminescent inks to print the s-modules instead of simple reflective-type ones guarantees the conventional notes aspect and its activation with such current standard procedures. This rationale behind the selection of inks that are luminescent under UV-standard radiation intended to render easier and cost-effective the implementation of these new s-modules QR codes, as it is in line with a well-established technology. Nonetheless, we note that the use of non-standard UV excitation may be tuned by the judicious choice of the ligands of the $\mathrm{Ln}^{3+}$-based organic complexes. Taking advantage of the $\mathrm{Ln}^{3+}$-based emission (intra4f transitions), pure red $\left(\mathrm{Eu}^{3+}\right)$ and green $\left(\mathrm{Tb}^{3+}\right)$ colours will be attained, independently of the ligands' sensitization energy ${ }^{31}$, This work is in line with the idea of selecting identification elements, through optical illumination, in different regions of the UV spectrum, thus, it has a huge potential to be implemented in parallel with well-established technology and procedures employed nowadays.

\section{Responsive QR codes}

Optimised luminescent QR codes based on s-modules were embossed on banknotes specimens for demonstration. The process used to multiplex the three levels of information combines the concept of $s$-modules and the RGB-based colour multiplexing ${ }^{21,22}$. Direct multiplexing can be achieved printing a black/white $\mathrm{QR}$ code and luminescent QR codes displaying emitting in the red (R) and green $(G)$ and eventually blue (not implemented in this work) spectral regions, provided by the dU6Eu and dU6Tb inks (see methods material synthesis), respectively. Dot shaped black module (denoted as $\downarrow$ ) is used for the base black/white QR code, providing public access information visible under daylight illumination and readable with any smartphone. Surrounding this module, two other modules characterised by different shapes (denoted as $\uparrow$ and $\equiv$ ) were built using two luminescent materials (as illustrated in the central column of Fig. 1), each one assign to a different accessibility level. The s-modules based methodology assisted by luminescent inks allow the construction of $\mathrm{QR}$ codes without superposition, avoiding colour mixture and assuring the separation and readability of the different levels.

To keep the QR code dimension analogous to that of a single black/white QR code, the s-module areas $\left(A_{i}\right)$ were optimised, minimizing the overall reading error probability $\left(R_{\text {error }}\right.$ Supplementary Eq. 1) associated to the misinterpretation of the module binary information (active or inactive). The overall $R_{\text {error }}$ was minimised as a function of the modules exposed areas using an unconstrained numerical method (Nelder-Mead) and yielding to the ratio between the module's areas $(\uparrow / \equiv / \downarrow)=1.00 / 0.77 / 0.43$ (Supplementary Fig. 13 and Supplementary information). Other regular polygon geometrical patterns may also be envisaged to build s-modules if the current detection limits of CCD cameras are pushed forward in the near future. This would certainly enable larger storage capacity s-modules (Supplementary Fig. 14, Supplementary Eq. 2 and Supplementary Information) up to eight times.

The optimised multiplexed QR codes were printed on banknote specimens, available from the European Central Bank, as an illustrative application example. The di-ureasil-based inks are amorphous and homogenous, resulting from the mixture at the molecular level of all the components (Supplementary Figs. 2-7 and Supplementary information). The number of printouts was optimised featuring luminescent QR codes with RGB pixel intensity values large enough to enable the colour demultiplexing using smartphones' CCD cameras. Although a single printout reveals remarkable intensity value, the saturation intensity of the $R$ and $G$ pixels occurs for a minimum number of printouts equal to 3 (dU6Eu) and 7 (dU6Tb), respectively, Fig. 2a. Hyperspectral microscopy shows that the emission colour is homogenous, at least, at the micrometre scale (Supplementary Figs. 6 and 7).

The first level of information is embedded in the black QR code module (public access) containing data about the exchange rate of the specimen facial value to other currencies, obtained on realtime from official data (X-Rates.com website). The second level of information is added displaying the information of the author's research affiliation institutions, using the luminescent $Q R$ codes for restrict access (green-emitting QR code, only accessed under UV radiation). The third level for combined restrict access and secure data transmission (red emitting QR code, accessed under UV radiation) contains an authentication tag (obtained with an AESCBC-MAC protocol) of the banknotes' serial number, which is a unique feature, allowing the individual identification of each specimen, Supplementary Table 3. This data is transmitted to a central server, via a secure connection, being used as a tag element employed to track the note transaction (see encrypting information for trackability section).

Under daylight illumination, the luminescent QR codes based on dU6Eu and dU6Tb inks are transparent enabling the typical use (public access, Fig. 1a) of the black/white QR code, Fig. 2b. Under UV, the emission is activated and the luminescent $Q R$ codes become visible, and a three-level multiplexed QR code is available, Fig. 2c, d, (restrict access, Fig. 1b). In particular, under UVA, the luminescent QR code reveals the R colour (Fig. 3b) from the dU6Eu that dominates over the G-based QR code (dU6Tb) that is, however, favoured under UVC irradiation, in whose scenario a bright green code is visible (Fig. 3c).

The transparency of the dU6Tb and dU6Eu inks guarantees the conventional notes aspect (Fig. 3a), as negligible absorption coefficient values, $a<10^{-4} \mathrm{~cm}^{-1}$ (Supplementary Fig. 11) in the vis ible spectral range, are detected. The absorption spectra in Fig. 2e are dominated by broad bands in the UV spectral region $(240-390 \mathrm{~nm})$, ascribed to the ligands singlet (S) excited states ${ }^{32-34}$ with large molar extinction coefficients $(\varepsilon)$ above $\sim 2 \times 10^{5} \mathrm{M}^{-1} \mathrm{~cm}^{-1}$ (Fig. 2e), analogous to those found in UVabsorbing organic dyes ${ }^{35}$, and quite large for $\mathrm{Ln}^{3+}$-based materials, enabling the excitation of a thin layer with commercial UV-emitting LEDs, converting it from transparent to bright $\mathrm{R}$ or $\mathrm{G}$ luminescent $\mathrm{QR}$ codes. The overlap between the absorption spectra of dU6Tb and dU6Eu enables that a single UV illumination source may be used to activate both luminescent QR codes. Nevertheless, we noticed that the absorption maximum peak position for each material is tuned to allow the selective and relative intensity favouring of the luminescent $Q R$ codes with $R$ and $G$ emission. Whereas the $R$ intensity is favoured under illumination at 275 and $350 \mathrm{~nm}$, the $\mathrm{G}$ one is maximised around 240 and $300 \mathrm{~nm}$.

The QR codes emission colour arises from the ${ }^{5} D_{4} \rightarrow{ }^{7} F_{6-3}$ (dU6Tb) and ${ }^{5} \mathrm{D}_{0} \rightarrow{ }^{7} \mathrm{~F}_{0-4}$ (dU6Eu) transitions, Fig. 2f. The low fullwidth-at-half-maximum ( $\mathrm{fwhm}<10 \mathrm{~nm}$ ) and the higher relative intensity of the ${ }^{5} D_{0} \rightarrow{ }^{7} F_{2}$ and ${ }^{5} D_{4} \rightarrow{ }^{7} F_{5}$ transitions peaking around $612 \mathrm{~nm}$ and $544 \mathrm{~nm}$, respectively, render the emission 
colour close to pure $\mathrm{G}$ and $\mathrm{R}$ colours, as illustrated in the Commission Internationale d'Éclairage (CIE) colour diagram in Fig. $2 \mathrm{~g}$. The near-pure colour emission is advantageous in what concerns the multiplexing analysis, as above detailed. The activation range of the $\mathrm{QR}$ codes emission was inferred from the selective excitation spectra monitored within the ${ }^{5} D_{0} \rightarrow{ }^{7} F_{2}$ and ${ }^{5} \mathrm{D}_{4} \rightarrow{ }^{7} \mathrm{~F}_{5}$ transitions for the dU6Eu and dU6Tb inks, respectively (Supplementary Figs. 8a and 9a). The excitation spectra resemble the absorption ones (Fig. 2e), pointing out that the main excitation mechanism is based on the ligands' sensitization process, rather than direct intra- $4 \mathrm{f}$ excitation. The population of the $\mathrm{Ln}^{3+}$ excited states mainly through ligands sensitization (low-lying triplet excited states, $T_{1}$ ) is advantageous because of the large $\varepsilon$ values (Fig. 2e) and the large ligands-induced Stokes shift, ensuring that the materials although emitting in the visible spectral range remain transparent under daylight conditions with negligible

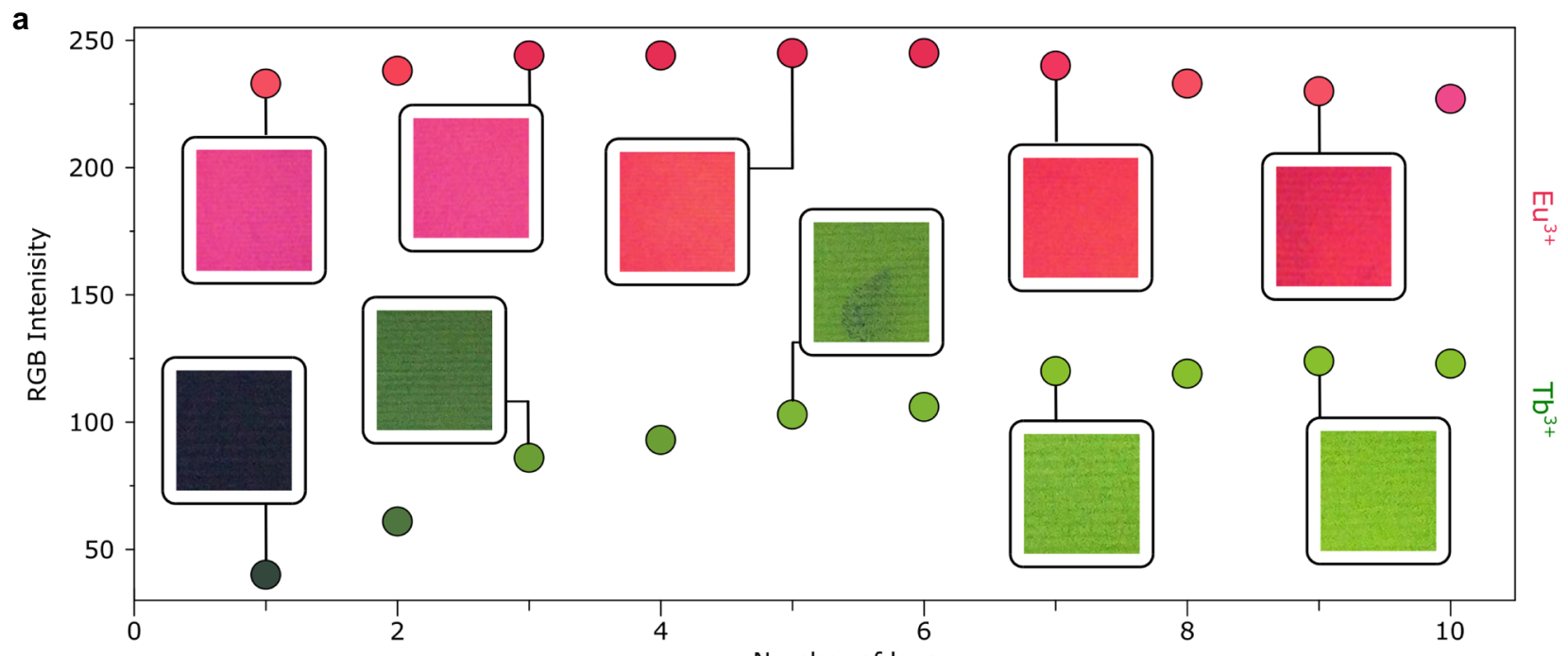

b
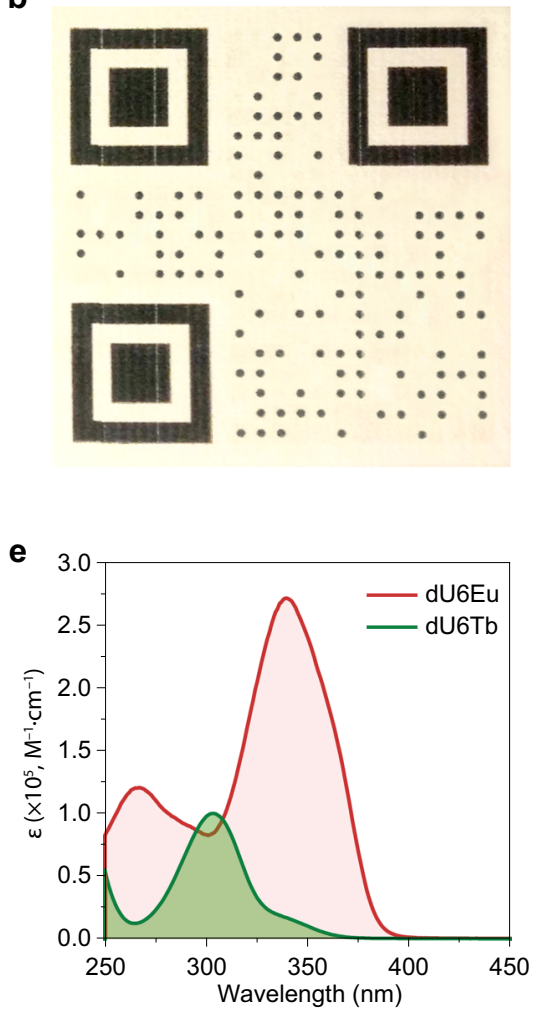
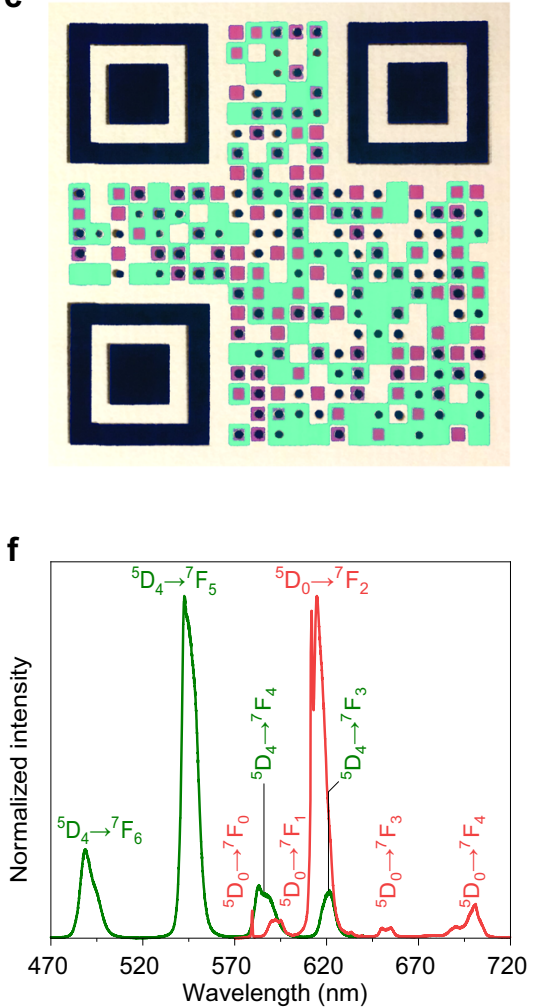

d

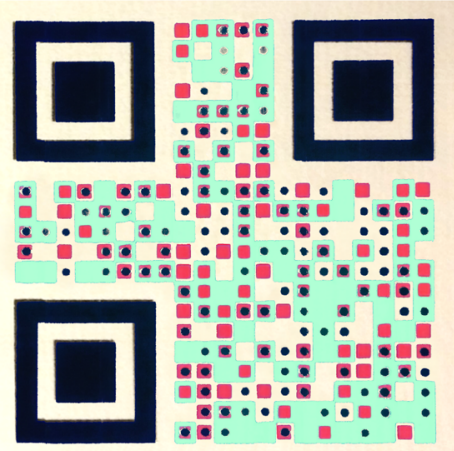

g

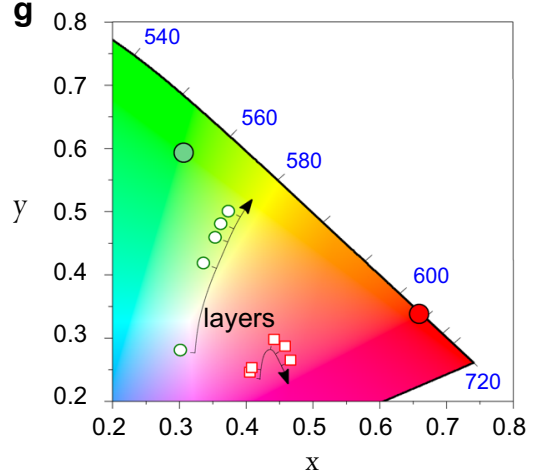

Fig. 2 Emission properties, printing and colour analyses. a Emitted intensity quantified by the RGB colour coordinates for dU6Tb as a function of the number of printouts under $254 \mathrm{~nm}$ excitation and for dU6Eu under $365 \mathrm{~nm}$ excitation. Images were obtained with a smartphone camera. The printed luminescent QR code images under $\mathbf{b}$ daylight excitation, $\mathbf{c} 254 \mathrm{~nm}$ excitation presenting an intense green colour and $\mathbf{d} 365 \mathrm{~nm}$ emitting a bright red colour. e Absorption extinction coefficient and $\mathbf{f}$ emission spectra excited at 350 and $370 \mathrm{~nm}$ for dU6Tb and dU6Eu, respectively. $g$ CIE diagram with the colour coordinates for dU6Eu and dU6Tb calculated from the emission spectra and with the colour coordinates for the images in (a) calculated from their RGB colour coordinates. 


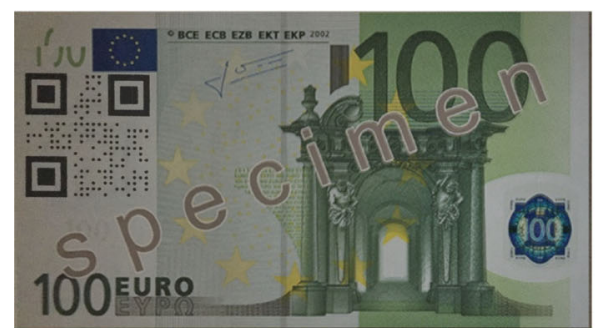

b
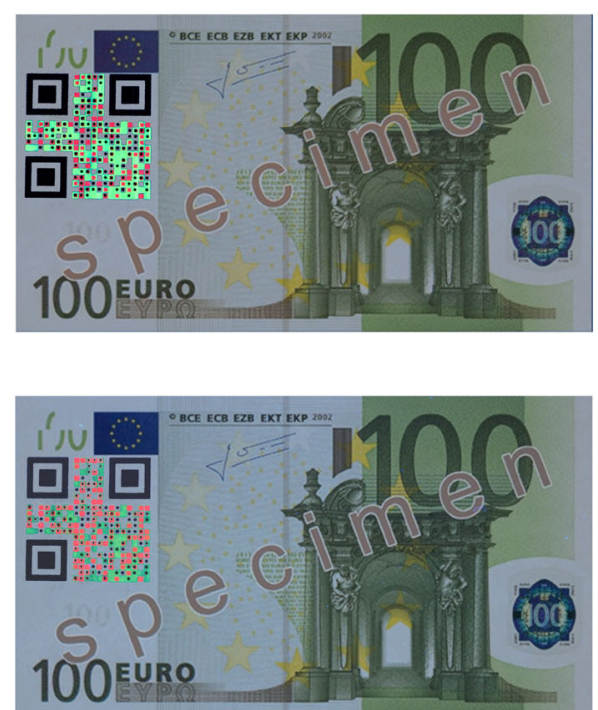

d
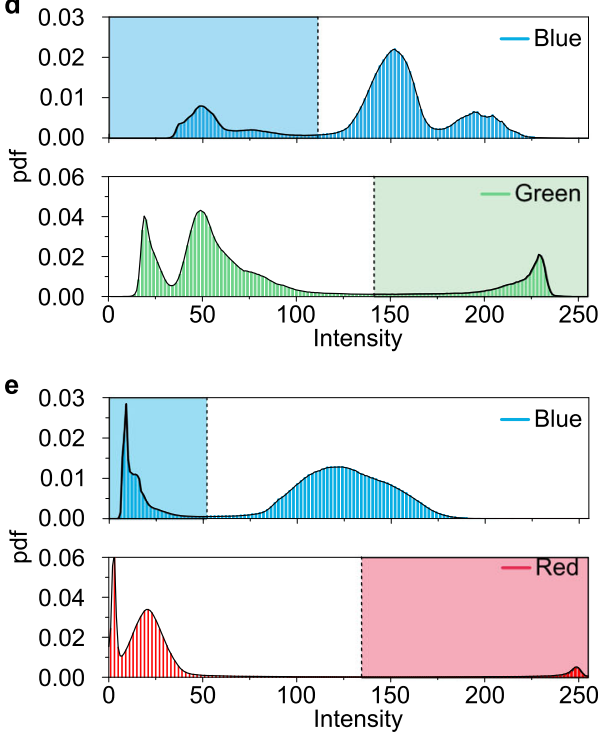

f

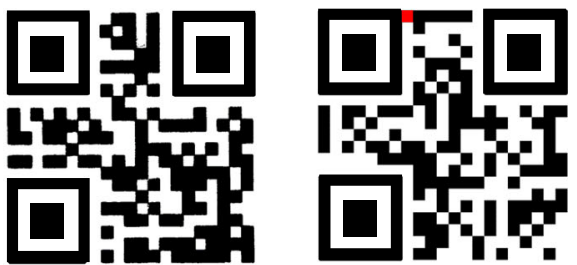

Fig. 3 Multiplexed luminescent QR code based on s-modules printed on banknote specimens. a Black QR code printed directly on top of a $100 €$ banknote specimen under daylight illumination. The QR code is fully visible and ready to be accessed. $\mathbf{b}$, $\mathbf{c}$ Images of the banknotes under $254 \mathrm{~nm}$ and $365 \mathrm{~nm}$ excitation, respectively, displaying the $\mathrm{Tb}^{3+}$ based QR code (green) and Eu ${ }^{3+}$ based QR code (red). d Blue and green coordinates histogram and e blue and red coordinates histogram for QR codes displayed in (b) and (c), respectively, with the decision level marked. The shaded areas are the modules colour coordinates of interest for identification. $f$ Decoded QR codes obtained from the decision level of (d) and (e). The red square in (f) is one misinterpreted module. The images in a-c are not real images; they are digital montages; whose only purpose is to illustrate a possible application.

self-absorption losses. Although direct hybrid-to- $\mathrm{Ln}^{3+}$ energy transfer involving the di-ureasil singlet $\left(S_{1}^{H}\right)$ and triplet $\left(T_{1}^{H}\right)$ excited states cannot be neglected (Supplementary Figs. 8 and 9), theoretical calculations demonstrated that ligand-to- $\mathrm{Ln}^{3+}$ energy transfer rates are one order of magnitude larger ${ }^{36}$. The main dominant intramolecular energy transfer pathway involving the $\mathrm{Ln}^{3+}$ levels is $\mathrm{S}_{1}^{H} \rightarrow T_{1}^{H} \rightarrow T_{1} \rightarrow{ }^{5} \mathrm{D}_{\mathrm{J}} \rightarrow{ }^{7} \mathrm{~F}_{\mathrm{J}^{\prime}}\left(\mathrm{J}=4, \mathrm{~J}^{\prime}=6-0\right.$ and $\mathrm{J}=0,1$, $\mathrm{J}^{\prime}=0-6$, for $\mathrm{Ln}^{3+}=\mathrm{Tb}^{3+}$ and $\mathrm{Eu}^{3+}$, respectively) ${ }^{23,34}$. We notice that, apart from a solvatochromic shift observed in the ligands' excited states, the optical properties of the inks are identical before and after drying (Supplementary Figs. 8 and 9) as pointed out by the structural results.

The emission properties were further quantified by the measurement of the absolute emission quantum yield, whose larger values (up to 0.40 , Supplementary Table 2) permits to predict the activation of the QR codes even at lower-excitation powers typical of commercial LEDs. The light emission efficiency and light-harvesting ability can be further related by the molar brightness $(B)$, given by $B=\varepsilon \times q^{37}$ allowing both properties to be compared across distinct samples. The $B$ values $\left(5.8 \times 10^{4}\right.$ and $3.3 \times 10^{4} \mathrm{M}^{-1} \mathrm{~cm}^{-1}$ for dU6Eu at $365 \mathrm{~nm}$ and dU6Tb at $315 \mathrm{~nm}$, respectively) are larger than the best values reported for other organic-dye molecules, with light emission in the $530-650 \mathrm{~nm}$ range $^{38-40}$.

Aiming at studying the role of moisture, and temperature on the QR codes stability, the emission quantum yield was monitored after placing the dry inks in a climatic chamber. In particular, the absolute emission quantum yield was evaluated upon severe accelerated ageing tests performed under controlled relative humidity (RH) and temperature (Supplementary Table 2). After 2 consecutive cycles of 8 and $16 \mathrm{~h}$ each $\left(40^{\circ} \mathrm{C}\right.$ and $\left.\mathrm{RH}=50 \%\right)$, only minor changes in the quantum yield values were recorded for the dU6Eu, whereas no change was measurable for dU6Tb. No variation was further detected after keeping the samples under ambient conditions $\left(\mathrm{T}=25^{\circ} \mathrm{C}, \mathrm{RH}=85 \%\right)$. The stability of emission colour was also studied after prolonged continuous solar irradiation (AM1.5G, $1000 \mathrm{Wm}^{-2}$ ). Despite a negligible intensity decrease found only for the red-related coordinate of the dU6Eu (Supplementary Fig. 12), the red and green colours remain detectable, using the smartphone CCD camera. Those results reinforce the active role of the di-ureasil organic-inorganic hybrids that enable the easy and tailored processing and simultaneously overcome the photodegradation ${ }^{31,41,42}$.

The black/white QR code can be decoded using a common application, therefore any further analysis or processing is avoided. For the luminescent QR codes, it is necessary to separate the different QR codes levels and proceed with decoding (Supplementary Fig. 15). A 'maximum-likelihood' criterion (Supplementary Information), was used to split both luminescent QR codes, based on a pixel colour identification, using a separation threshold level (see methods Colour identification process). The probability density function (pdf) for the pixels' intensity distribution (defined in an 8-bit dimension) in the 3 colour space channels $(R, G, B)$ was calculated from the photos of the embedded codes (Fig. 3b, c). The red and green channels combined with the blue one (Fig. 3d, e) were used to retrieve the luminescent QR codes 
based on dU6Eu and dU6Tb, respectively. From this method, it is possible to extract the predicted probability error $\left(P_{\text {error }}\right)$, obtained through the calculus of the pdf integral. For further comparison (Supplementary Table 4), it is also presented the experimental error $\left(E_{\text {error }}\right)$ defined as the number of modules whose colour was misinterpreted, resulting in $E_{\text {error }}<0.1 \%$. As the embedded forward error correction code can handle (and correct) interpretation error rates up to $30 \%$, this $E_{\text {error }}$ can be considered error-free. The red $\mathrm{QR}$ code, decoded using a dedicated free $\mathrm{QR}$ code reader application, contains encrypted data which must be transmitted to a central server, as described next.

\section{Encrypting information for trackability}

A system that enforces security and trackability relies on two factors: (a) secure encoding of the information/serial number in the QR code and (b) secure transmission of the tag information to a server. Besides the security imposed by the UV excitation granted accessibility, additional security features can be explored. A new and relevant level of security is added with the inclusion of smodules QR code. Contrarily to the passive and static/immutable conventional security elements, those QR codes are active and dynamic tags. Taking advantage of the colour space orthogonality, assisted by spatial multiplexing, it is possible to increase the capacity of QR codes and encode (using one single colour) the note serial number using a secure block-cipher (Advanced Encryption Standard AES) with 128 bits. This procedure ensures that each note has a unique identification tag and that it is not possible to create new valid authentication tags for each serial number. Each time a transaction occurs, the encrypted identification information is transmitted, in a secure way to a central server, encrypted with RSA-4096 bits, allowing this way tracking the usage of the banknotes. In summary, the security is not achieved by the inability to read the QR code, but rather in its ability to prevent the creation of valid forged (new) tags and on the security of the ciphers used for the communication. With respect to long-term security, AES-128 and RSA-4096 are solutions resilient to classical attacks, and according to NIST recommended for usage beyond $2030^{43}$.

The ISO standard for QR codes allows the encoding of strings of length 25 over a 45-letter alphabet resulting in a maximum of 137 bits of information, $\log _{2}\left(45^{25}\right)$. This size is large enough to contain a Message Authentication Code (MAC) produced with AES-128 (128 bits), resulting in the possibility of securely encoding any message with 128 bits into a QR code such as our example of banknotes, that only needs 42 bits ( 1 capital letter +11 numbers, $\left.\log _{2}\left(26 \times 10^{11}\right)\right)$. The identifying serial number of each note was thus encrypted into a 128-bit string unique to each note. More precisely, we encode the serial number of each note in 42 bits, pad this message to 128 bits, apply CBC-MAC to it (AES-128), and encode the resulting cipher (also 128 bits) in a QR code of length 25 (string presented in Supplementary Table 1).

The cipher block chaining Message Authentication Code (CBC$M A C)$ construction is secure for fixed-length messages given the security of the underlying block-cipher (AES), under the standard security notion for MACs, resistance under existential forgery for adaptive chosen-message attacks ${ }^{44,45}$. This security notion states that an adversary cannot forge new valid pairs (message, authentication tag) for messages he has not seen before. In our case, it means that it is possible to clone a known QR code, but it is not possible to forge/create valid new QR codes.

After retrieving the string encoded in the QR code containing the encrypted note serial number, the client application establishes a secure connection with the server. In this communication, the client transmits to the server the QR code information, its geographic location and a timestamp to ensure freshness encrypted with RSA-4096 bits. On receiving this ciphertext the server decrypts it, validates the integrity of the received message, and checks if the elapsed time since the timestamp is less than a preconfigured value to avoid a replay of the same transaction. The server then stores information such as date of the note usage, the geographic location (e.g. based on the IP address) where the note was transacted, and (eventually) provides a transaction warning message to the client application, regarding the authentication of the note (or if it is on a blacklist), as illustrated in Fig. 4.

After establishing the concept of multiplexed QR codes based on $s$-modules as smart labels for trackability and loT, a practical example for decoding was performed through the development of free access and user-friendly mobile app for smartphones (Supplementary Fig. 16 and Supplementary Information). This app allows to decode the luminescent multiplexed QR code, presenting the public information to the user and when UV-excited retrieves the restrict access. Simultaneously, it establishes a secure connection to a server sending the encrypted information to be stored (more details in Supplementary Information). This app in conjugation with luminescent responsive banknote prototypes is unequivocally a new generation of stamps that combine authentication, security features and loT. The app for IOS smartphones is free and available for download at https://tinyurl.com/QR-note.

\section{DISCUSSION}

We demonstrated a single multiplexed QR code based on supermodules (s-modules) with enhanced storage capacity (triple than that of a traditional black/white QR code with the same dimensions) printed with flexible luminescent inks based on organic-inorganic materials modified by $\mathrm{Ln}^{3+}$ ions with tunable colour in the RGB colour space. The hybrid materials are prepared through a well-studied and optimised process near ambient temperature using low-cost precursors and non-toxic solvents being promising inks in a scale-up scenario for industrial applications. The multiplexed QR code size was optimised based on the use of s-modules formed by modules with distinct shapes (squares and circles) and different areas, baring the same location patterns, for considering a typical reading distance $(0.20 \mathrm{~m})$. The optimised dimensions yield a QR code with $4.5 \times 4.5 \mathrm{~cm}^{2}$ that fits most of the current applications and ensures detection and reading. Moreover, we also demonstrated a real application using banknotes combining loT applications with restrict and encrypted access. The black/white QR code confers to the banknote societal loT interaction and the luminescent part of the multiplexed QR code is devoted to restrict access. The green and red parts of the multiplexed QR code are UV-activated in analogous conditions to that used nowadays for banknote security track. The secure access was further demonstrated using the red-emitting modules QR code through encrypted information. The high-efficiency and pure colours emission from the $\mathrm{Ln}^{3+}$-based inks ensures the multiplexed QR code readability under the illumination of commercial LEDs and under natural daylight demonstrating that active QR codes, for smart trackability and loT, could be realised using the concept of $s$-modules multiplexed in colour. Featuring the detection limits of CCD cameras in a near future, s-modules with storage capacity up to $8 \times$ may be envisaged by tuning the spatial multiplexing by simply adding to the circles and squares modules other regular polygon geometrical patterns.

\section{METHODS}

Material synthesis

The di-ureasils' synthesis protocol is a well-studied and optimised process, so that materials replication can be easily attained ${ }^{46,47}$, despite processing by inkjet printing is a new aspect that potentiates other applications besides the traditional monolithic and film deposition processing. The diureasil $\mathrm{d}-\mathrm{U}(600)$, formed by polyether chains (average molecular weight of $600 \mathrm{~g} \cdot \mathrm{mol}^{-1}$ ) covalently linked to a siliceous inorganic skeleton by urea bridges, was prepared according to the literature ${ }^{46,47}$. The di-ureasil hybrid was modified by $\mathrm{Eu}^{3+}$ - and $\mathrm{Tb}^{3+}$-based complexes, as detailed in 


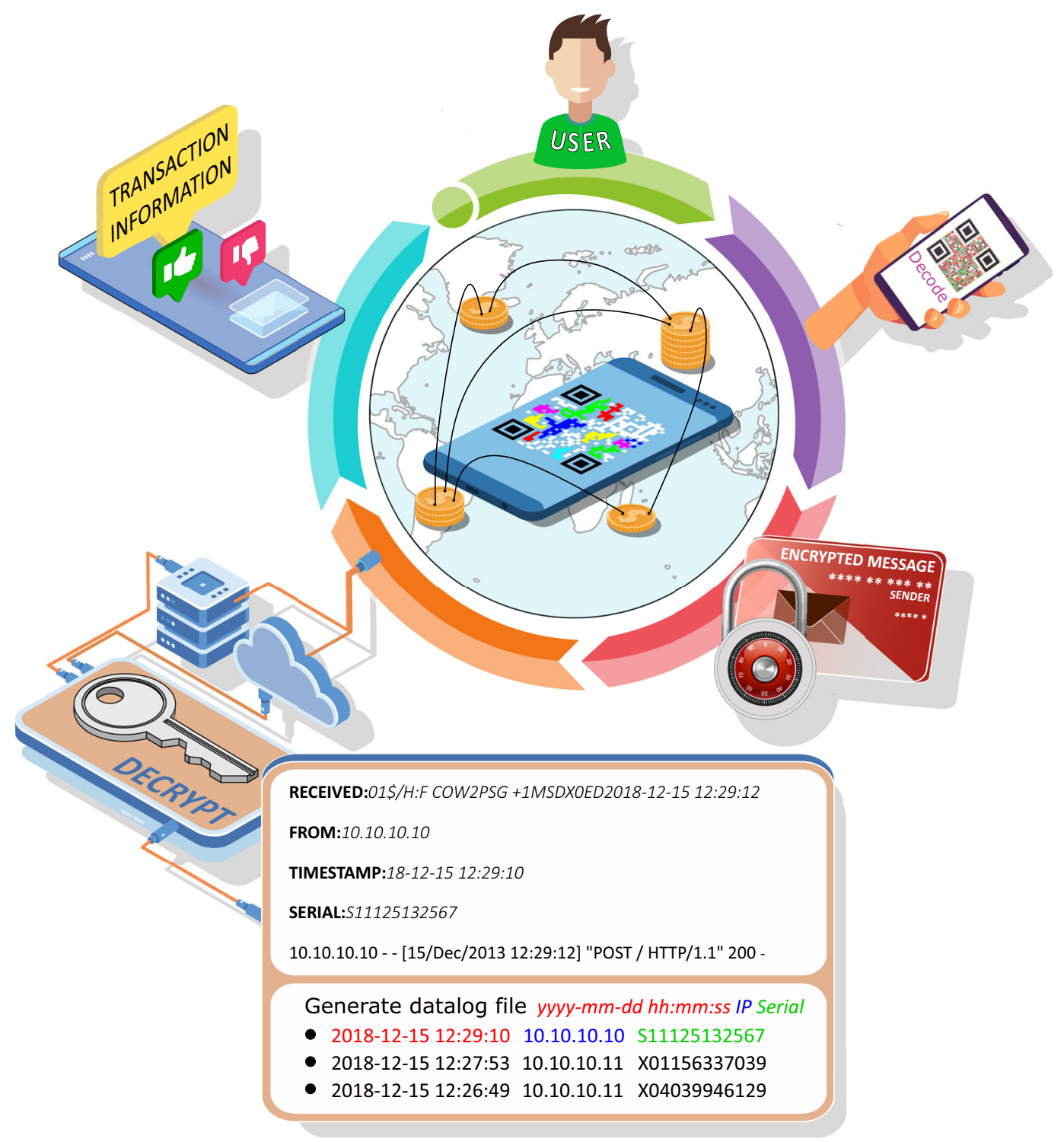

Fig. 4 Transaction process diagram. The QR code is decoded and the encrypted information is sent to a server/cloud service that has the key to decrypt the received information. An alert can be trigged with the transaction information and transmitting to the client app.

Supplementary Information. The structures of non-hydrolyzed organicinorganic hybrid precursor, and of the complexes are illustrated in Supplementary Fig. 1. Supplementary Information contains details of structural (X-ray diffraction, scanning electron microscopy with energydispersive X-ray, Fourier transform infrared (FT-IR) spectroscopy) and optical characterisation (photoluminescence and hyperspectral imaging) of the fabricated materials, Supplementary Figs. 2-11. To enable the materials processing as inks to be printed in a commercial inkjet printer, the viscosity was controlled and measured using a Brookfield DV- II+Pro viscometer using the Enhanced Brookfield UL Adapter. The viscosity values were set as $5.02 \mathrm{CP}$ and $2.34 \mathrm{CP}$ (at $20^{\circ} \mathrm{C}$ ) for dU6Eu and dU6Tb, respectively.

\section{Printing process}

The multiplexed QR code was printed with dimensions $4.5 \times 4.5 \mathrm{~cm}^{2}$ in order to fit within the banknote and to ensure reading, considering an optimised distance between the CCD camera of the smartphone and the QR code $(0.2 \mathrm{~m}$, Supplementary Fig. 14 and more details in Supplementary Information). The first access level modules, with a dot-shaped, was printed with black colour, using a commercial laser printer (Xerox Colour C60) over Ingres from Fabriano Inc. paper (worldwide producer of banknote paper). It is an uncoated laid paper made with elemental chlorine-free pulp with $90 \pm 3 \% \mathrm{~g} / \mathrm{m}^{2}$ (ISO 536), opacity $90 \pm 3 \%$ (ISO 2471) and roughness $1100 \pm 300 \mathrm{~mL} / \mathrm{min}$ (ISO 8791-2) according to the manufacturer. The luminescent QR codes were printed using the dU6Eu and dU6Tb inks in a commercial inkjet printer (Brother DCP - J562DW), with a resolution of $6000 \times 1200$ dots per inch, able to produce 12 black or 6 colour copies per minute (ISO/IEC 29183) with drop size $\sim 1.5 \mathrm{pL}$. All the QR codes were generated using online software (https://www.qrcode-monkey. comwebsite) and the printing followed the legislation for the use of specimens printed money.

\section{Image processing}

The images of the luminescent QR codes under daylight and UV illumination were captured with a smartphone camera with a resolution of $4160 \times 3120$ pixel $^{2}$, aperture of $f / 1.9$ and sensor dimension of $1 / 2.6 "$. The photographs of the $\mathrm{QR}$ codes superimposed on banknotes specimens were taken using a Canon 5D Mkll with a lens Super Macro-Takumar $50 \mathrm{~mm} \mathrm{~F} 4$ and an extension tube of $28 \mathrm{~mm}$. The UV excitation sources were a portable lamp (Spectroline ENF-280C/FE and Shanghai Heqin Analytical Instruments Co. Ltd High-Intensity Ultraviolet Analyzer UV-3000) and UV-emitting LEDs (Ocean Optics MCLS LLS-365).

\section{Optical properties}

UV/Visible spectroscopy: UV/visible absorption spectra were measured using a Lambda 950 dual-beam spectrometer (Perkin-Elmer). All measurements 
were performed using a $1 \mathrm{~cm}$ optical path quartz cell. Using the BeerLambert law, the absorption coefficient $\left(a, \mathrm{~cm}^{-1}\right)$ and the molar extinction coefficient $\left(\varepsilon, \mathrm{M}^{-1} \mathrm{~cm}^{-1}\right)$ were calculated.

Photoluminescence spectroscopy. The photoluminescence spectra were recorded at room temperature with a modular double-grating excitation spectrofluorimeter with a TRIAX 320 emission monochromator (Fluorolog3. Horiba Scientific) coupled to an R928 Hamamatsu photomultiplier. The emission spectra of the luminescent $\mathrm{QR}$ codes were measured using a spectrometer OceanOptics Maya 2000 Pro coupled with an optical fibre, under UV excitation ( $365 \mathrm{~nm}$ ).

Absolute emission quantum yield. The absolute emission quantum yield values (q) were measured at room temperature using a C9920-02 Hamamatsu system. The method is accurate within $10 \%$.

\section{Colour demultiplexing methodology}

The multiplexed QR code photograph (Supplementary Fig. 15a) is split into the three RGB colour space channels, Supplementary Fig. 15. Then, for each channel the intensity pdf (Supplementary Eq. 3) is calculated in the range $[0,256]$ (Supplementary Fig. 15c); being described by Gaussian distribution functions. The presence of more than one discriminated Gaussian distribution function in each channel indicates that the channel' information can be used to separate the modules by colours $^{22}$. The optimal decision level was established by minimizing the error pdf (Supplementary Eqs. 4 and 5) and used as a threshold value to separate the different colour modules, Supplementary Fig. $15 \mathrm{~d}$. The image was split into $21 \times 21$ modules $^{2}$ where the contiguous regions were mapped and grouped according to size. Afterwards, the original QR codes (Supplementary Fig. $15 \mathrm{e})$ are ready to be decoded and the access to server/cloud established.

\section{DATA AVAILABILITY}

The authors declare that the main data supporting the findings of this study are available within the article and its Supplementary Information files. Extra data are available from the corresponding author upon request.

Received: 26 November 2019; Accepted: 23 April 2020;

Published online: 22 June 2020

\section{REFERENCES}

1. Wang, K. et al. Green industrial Internet of Things architecture: an energy-efficient perspective. IEEE Commun. Mag. 54, 48-54 (2016).

2. Zanella, A. et al. Internet of Things for smart cities. IEEE Internet Things 1, 22-32 (2014).

3. Akyildiz, I. F. \& Jornet, J. M. The Internet of nano-things. IEEE Wirel. Commun. 17, 58-63 (2010).

4. Holden, N. M., White, E. P., Lange, M. C. \& Oldfield, T. L. Review of the sustainability of food systems and transition using the Internet of Food. npj Sci. Food 2, 18 (2018).

5. Goldsmith, B. R. et al. Digital biosensing by foundry-fabricated graphene sensors. Sci. Rep. UK 9, 434 (2019).

6. Curry, A. The internet of animals. Nature 562, 322-326 (2018).

7. Kleineberg, K. K. \& Helbing, D. Collective navigation of complex networks: participatory greedy routing. Sci. Rep. UK 7, 2897 (2017).

8. Gazis, V. A survey of standards for Machine-to-Machine and the Internet of Things. IEEE Commun. Surv. Tut. 19, 482-511 (2017).

9. Sun, Y. C., Song, H. B., Jara, A. J. \& Bie, R. F. Internet of Things and big data analytics for smart and connected communities. IEEE Access 4, 766-773 (2016).

10. Gubbi, J., Buyya, R., Marusic, S. \& Palaniswami, M. Internet of Things (loT): a vision, architectural elements, and future directions. Future Gener. Comp. Syst. 29, 1645-1660 (2013).

11. Kanikar, K.P., Singhr, S. \& Guptar, B. K. A novel approach to synthesise a dualmode luminescent composite pigment for uncloneable high-security codes to combat counterfeiting. Chem. Eur. J. 23, 17144-17151 (2017).

12. Kumar, P., Singh, S. \& Gupta, B. K. Future prospects of luminescent nanomaterial based security inks: from synthesis to anti-counterfeiting applications. Nanoscale 8, 14297-14340 (2016).

13. Al-Fuqaha, A. et al. Internet of Things: a survey on enabling technologies, protocols, and applications. IEEE Commun. Surv. Tut. 17, 2347-2376 (2015).
14. Woodland, N. J. \& Bernard, S., Classifying apparatus and method, US2612994, 1952.

15. Meruga, J. M. et al. Red-green-blue printing using luminescence-upconversion inks. J. Mater. Chem. C. 2, 2221-2227 (2014).

16. Sangeetha, N. M. et al. 3D assembly of upconverting $\mathrm{NaYF}_{4}$ nanocrystals by AFM nanoxerography: creation of anti-counterfeiting microtags. Nanoscale 5, 9587-9592 (2013)

17. Diaz, R. et al. High-throughput fabrication of anticounterfeiting colloid-based photoluminescent microtags using electrical nanoimprint lithography. Nanotechnology 25, 345302 (2014).

18. Gangwar, A. K., Nagpal, K. \& Gupta, B. K. Triluminescent functional composite pigment for non-replicable security codes to combat counterfeiting. Chemistryselect 3, 9627-9633 (2018).

19. Gangwar, A. K. et al. Single excitable dual emissive novel luminescent pigment to generate advanced security features for anti-counterfeiting applications. J. Mater. Chem. C. 7, 13867-13877 (2019).

20. Singh, A. K., Singh, S. \& Gupta, B. K. Highly efficient, chemically stable, and UV/ blue-light-excitable biluminescent security ink to combat counterfeiting. ACS Appl. Mater. Interfaces 10, 44570-44575 (2018).

21. André, P. S. \& Ferreira, R. A. S. Colour multiplexing of quick-response (QR) codes. Electron. Lett. 50, 1828-1830 (2014).

22. Ramalho, J. F. C. B. et al. Luminescent QR codes for smart labelling and sensing. Opt. Laser Technol. 101, 304-311 (2018).

23. Ramalho, J. F. C. B. et al. Luminescence thermometry on the route of the mobilebased Internet of Things (IoT): how smart QR codes make it real. Adv. Sci. 6, 1900950 (2019).

24. Kumar, P., Nagpal, K. \& Gupta, B. K. Unclonable security codes designed from multicolor luminescent lanthanide-doped $\mathrm{Y} 2 \mathrm{O} 3$ nanorods for anticounterfeiting. ACS Appl. Mater. Interfaces 9, 14301-14308 (2017).

25. Carlos, L. D. et al. Full-color phosphors from Europium (III)-based organosilicates. Adv. Mater. 12, 594-598 (2000).

26. Sanchez, C., Lebeau, B., Chaput, F. \& Boilot, J. P. Optical properties of functional hybrid organic-inorganic nanocomposites. Adv. Mater. 15, 1969-1994 (2003).

27. Parola, S., Julian-Lopez, B., Carlos, L. D. \& Sanchez, C. Optical properties of hybrid organic-inorganic materials and their applications. Adv. Funct. Mater. 26, 6506-6544 (2016).

28. Willis-Fox, N. et al. Tunable white-light emission from conjugated polymer-diureasil materials. Adv. Funct. Mater. 26, 532-542 (2016).

29. Binnemans, K. Rare-earth beta-diketonates. Handbook on the physics and chemistry of rare Earths 35 (107-272. Elsevier, Amsterdam, 2005).

30. Fairchild, M. D. Book color appearance models (John Wiley \& Sons Ltd, England, 2005).

31. Carlos, L. D., Ferreira, R. A. S., de Zea Bermudez, V. \& Ribeiro, S. J. L. Lanthanidecontaining light-emitting organic-inorganic hybrids: a bet on the future. Adv. Mater. 21, 509-534 (2009).

32. Dwivedi, Y., Singh, A. K., Prakash, R. \& Rai, S. B. Preparation and characterization of $\mathrm{Tb}^{3+}$ and $\mathrm{Tb}(\mathrm{sal})_{3}$ center dot $\mathrm{nH}_{2} \mathrm{O}$ doped PC:PMMA blend. J. Lumin. 131, 2451-2456 (2011).

33. Shen, Z. H. et al. Synthesis, characterization, and biological activity of some lanthanide ternary complexes. J. Coord. Chem. 64, 2342-2352 (2011).

34. Fang, $\mathrm{M}$. et al. Efficient green-emitting $\mathrm{Tb}^{3+}$-doped di-ureasil coating phosphors for near-UV excited light-emitting diodes. J. Lumin. 219, 116910 (2020).

35. Lian, X. H., Wei, M. Y. \& Ma, Q. Nanomedicines for near-infrared fluorescent lifetime-based bioimaging. Front. Bioeng. Biotech. 7, 386 (2019).

36. Lima, P. P. et al. Energy transfer mechanisms in organic-inorganic hybrids incorporating Europium(III): a quantitative assessment by light emission spectroscopy. J. Phys. Chem. C. 111, 17627-17634 (2007).

37. Reineck, P. et al. Brightness and photostability of emerging red and near-IR fluorescent nanomaterials for bioimaging. Adv. Opt. Mater. 4, 1549-1557 (2016).

38. Frias, A. R. et al. Sustainable luminescent solar concentrators based on organicinorganic hybrids modified with chlorophyll. J. Mater. Chem. A 6, 8712-8723 (2018).

39. Heim, R. \& Tsien, R. Y. Engineering green fluorescent protein for improved brightness, longer wavelengths and fluorescence resonance energy transfer. Curr. Biol. 6, 178-182 (1996).

40. Butkevich, A. N. et al. Hydroxylated fluorescent dyes for live-cell labeling: synthesis, spectra and super-resolution STED microscopy. Chem. Eur. J. 23, 12114-12119 (2017).

41. Lima, P. P. et al. Spectroscopic study of a UV-photostable organic-inorganic hybrids incorporating an $\mathrm{Eu}^{3+}$ beta-diketonate complex. ChemPhysChem 7, 735-746 (2006). 
42. Lima, P. P. et al. Photo-click chemistry to design highly efficient lanthanide betadiketonate complexes stable under UV irradiation. Chem. Mater. 25, 586-598 (2013).

43. Silverman, R. D. Has the RSA algorithm been compromised as a result of Bernstein's paper? (RSA Laboratories, 2002).

44. Bellare, M., Kilian, J. \& P. The security of the cipher block chaining message authentication code. J. Comput. Syst. Sci. 61, 362-399 (2000).

45. Goldwasser, S., Micali, S. \& Rivest, R. L. A digital signature scheme secure against adaptive chosen-message attacks. SIAM J. Comput. 17, 281-308 (1988).

46. Carlos, L. D. et al. Sol-gel derived urea cross-linked organically modified silicates. 2. Blue-light emission. Chem. Mater. 11, 581-588 (1999).

47. Armand, M., Poinsignon, C. J., Sanchez, J.-Y. \& de Zea Bermudez, V. French Patent 91 11349, 1991

\section{ACKNOWLEDGEMENTS}

This work was developed within the scope of the project CICECO-Aveiro Institute of Materials (UIDB/50011/2020 \& UIDP/50011/2020), Instituto de Telecomunicações (FCT Ref. UIDB/50008/2020-UIDP/50008/2020), WinLEDs (POCI-01-0145-FEDER-030351) and Graphsense (POCl-01-0145-FEDER-032072) financed by national funds through the FCT/MEC and when appropriate co-financed by FEDER under the PT2020 Partnership through European Regional Development Fund (ERDF) in the frame of Operational Competitiveness and Internationalization Programme ( $\mathrm{POCl})$. SFHC thanks Solar-Flex, CENTRO-01-0145-FEDER-030186. A.M. Botas and M. Ferro from the University of Aveiro are acknowledged for help in the hyperspectral and scanning electronic microscopies, respectively.

\section{AUTHOR CONTRIBUTIONS}

P.S.A. and R.A.S.F. conceived the idea of the experiment. Experimental data were taken by J.F.C.B.R., L.M.S.D., and S.F.H.C. The synthesis and processing were carried out by L.F., S.F.H.C. and J.F.C.B.R. The app was developed by P.S.A. and J.F.C.B.R., P.A. and P.M. carried out the information encrypting. P.S.A. and R.A.S.F. co-wrote the paper with input from all authors.

\section{COMPETING INTERESTS}

A patent application about luminescent QR codes spectrally and spatially multiplexed for real time trackability was filled by University of Aveiro and Instituto de Telecomunicações with J.F.C.B.R., R.A.S.F and P.S.A. as inventors, pending with application number PN115622 (Portugal). The remaining authors declare that there are no conflicts of interest related to this article.

\section{ADDITIONAL INFORMATION}

Supplementary information is available for this paper at https://doi.org/10.1038/ s41528-020-0073-1.

Correspondence and requests for materials should be addressed to R.A.S.F. or P.S.A.

Reprints and permission information is available at http://www.nature.com/ reprints

Publisher's note Springer Nature remains neutral with regard to jurisdictional claims in published maps and institutional affiliations.

\begin{abstract}
(c) (i)
Open Access This article is licensed under a Creative Commons Attribution 4.0 International License, which permits use, sharing, adaptation, distribution and reproduction in any medium or format, as long as you give appropriate credit to the original author(s) and the source, provide a link to the Creative Commons license, and indicate if changes were made. The images or other third party material in this article are included in the article's Creative Commons license, unless indicated otherwise in a credit line to the material. If material is not included in the article's Creative Commons license and your intended use is not permitted by statutory regulation or exceeds the permitted use, you will need to obtain permission directly from the copyright holder. To view a copy of this license, visit http://creativecommons. org/licenses/by/4.0/.
\end{abstract}

(c) The Author(s) 2020 